\title{
Open Subsets of LF-spaces
}

by

\author{
Kotaro MINE and Katsuro SAKAI
}

Presented by Stanistaw KWAPIEŃ

\begin{abstract}
Summary. Let $F=\operatorname{ind} \lim F_{n}$ be an infinite-dimensional LF-space with density dens $F=$ $\tau\left(\geq \aleph_{0}\right)$ such that some $F_{n}$ is infinite-dimensional and dens $F_{n}=\tau$. It is proved that every open subset of $F$ is homeomorphic to the product of an $\ell_{2}(\tau)$-manifold and $\mathbb{R}^{\infty}=$ ind $\lim \mathbb{R}^{n}$ (hence the product of an open subset of $\ell_{2}(\tau)$ and $\mathbb{R}^{\infty}$ ). As a consequence, any two open sets in $F$ are homeomorphic if they have the same homotopy type.
\end{abstract}

1. Introduction. A locally convex topological linear space $F$ is called an LF-space if it is the strict inductive limit of Fréchet spaces $\left({ }^{1}\right)$. More precisely, $F$ has a tower $F_{1} \subsetneq F_{2} \subsetneq \cdots$ of linear subspaces being Fréchet and a local basis consisting of balanced (circled) convex sets $V$ such that $V \cap F_{n}$ is a neighborhood of 0 in $F_{n}$ for each $n \in \mathbb{N}$. Then we write $F=\operatorname{ind} \lim F_{n}$. Given countably many Fréchet spaces $F_{n}, n \in \mathbb{N}$, we define $\sum_{n=1}^{\infty} F_{n}=$ ind $\lim \prod_{i=1}^{n} F_{i}$, where each $\prod_{i=1}^{n} F_{i}$ is identified with the subspace $\prod_{i=1}^{n} F_{i} \times$ $\{0\}$ of $\prod_{i=1}^{n+1} F_{i}$. For LF-spaces, we refer to [8, Ch. II, §6], [12, Ch. 13], etc.

In this paper, we also consider the (topological) direct limit of a tower $X_{1} \subset X_{2} \subset \cdots$ of (topological) spaces which is denoted by $\lim _{\longrightarrow} X_{n}$, that is, $\lim _{n} X_{n}=\bigcup_{n \in \mathbb{N}} X_{n}$ with the topology such that $U$ is open $\overrightarrow{\text { in }} X_{\underline{X}} X_{n}$ if and only if $U \cap X_{n}$ is open in $X_{n}$ for each $n \in \mathbb{N}$. Even if each $\vec{X}_{n}$ is a topological linear space, $\lim _{\longrightarrow} X_{n}$ is not in general. If the addition of $\lim _{\longrightarrow} X_{n}$ is continuous, then it is a topological linear space $\left({ }^{2}\right)$. In this case, if every $X_{n}$

2000 Mathematics Subject Classification: 46A13, 46T05, 57N17, 57N20.

Key words and phrases: LF-space, density, open set, direct limit, $\mathbb{R}^{\infty}, \ell_{2}(\tau)$-manifold, $\ell_{2}(\tau) \times \mathbb{R}^{\infty}$-manifold

This work is supported by Grant-in-Aid for Scientific Research (No. 17540061).

$\left({ }^{1}\right)$ A Fréchet space is a locally convex completely metrizable topological linear space.

$\left({ }^{2}\right)$ The scalar multiplication is continuous because $\mathbb{R} \times \lim _{\longrightarrow} X_{n}=\underset{\lim }{\longrightarrow}\left(\mathbb{R} \times X_{n}\right)$. However,

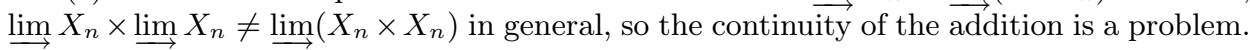


is locally convex then so is $\lim _{\longrightarrow} X_{n}$ (cf. [12, Problem 13-1-5]). For the tower $\mathbb{R} \subset \mathbb{R}^{2} \subset \mathbb{R}^{3} \subset \cdots$, the direct limit $\mathbb{R}^{\infty}=\lim \mathbb{R}^{n}$ is a topological linear space, hence $\mathbb{R}^{\infty}$ is an LF-space, i.e., $\mathbb{R}^{\infty}=\overrightarrow{\operatorname{ind}} \lim \mathbb{R}^{n}$. The Hilbert space with density $\tau\left(\geq \aleph_{0}\right)$ is denoted by $\ell_{2}(\tau)$, where $\ell_{2}=\ell_{2}\left(\aleph_{0}\right)$.

The topological classification problem for LF-spaces is completely solved by the results of Mankiewicz [6, Theorem 2.14] and Torunczyk [11, Theorem 6.1]: every LF-space $F=\operatorname{ind} \lim F_{n}$ is homeomorphic to $(\approx)$ one of the spaces $\mathbb{R}^{\infty}, \ell_{2}(\tau) \times \mathbb{R}^{\infty}$ or $\sum_{n=1}^{\infty} \ell_{2}\left(\tau_{n}\right)$, where $\tau=\operatorname{dens} F$ and $\tau_{1}<\tau_{2}<\cdots$ with $\sup \tau_{i}=\operatorname{dens} F$. In fact, (1) $F \approx \mathbb{R}^{\infty}$ if $\operatorname{dim} F_{n}<\infty$ for each $n \in \mathbb{N}$; (2) $F \approx \ell_{2}(\tau) \times \mathbb{R}^{\infty}$ if some $F_{n}$ is infinite-dimensional and dens $F_{n}=\operatorname{dens} F$ $=\tau$; (3) $F \approx \sum_{i=1}^{\infty} \ell_{2}\left(\tau_{i}\right)$ if dens $F_{n}<\operatorname{dens} F$ for every $n \in \mathbb{N}$.

Given a space $E$ (called a model space), a paracompact Hausdorff space $M$ is called an E-manifold if it is locally homeomorphic to $E$, that is, each point of $M$ has an open neighborhood homeomorphic to an open set in $E$. Although the theory of $\mathbb{R}^{\infty}$-manifolds has been well developed (cf. [3], [7], etc.), that of $\ell_{2} \times \mathbb{R}^{\infty}$-manifolds has not. Not much is known about $\ell_{2}(\tau) \times \mathbb{R}^{\infty}$ - or $\sum_{i=1}^{\infty} \ell_{2}\left(\tau_{i}\right)$-manifolds.

In the following,

let $F$ be an $L F$-space such that $F \approx \ell_{2}(\tau) \times \mathbb{R}^{\infty}$, where $\tau \geq \aleph_{0}$.

In this paper, we show the following:

Main Theorem. For each open set $U$ in $F$, there exists an $\ell_{2}(\tau)$-manifold $M$ such that $U \approx M \times \mathbb{R}^{\infty}$.

We have the following corollaries. The first one follows from the classification theorem for $\ell_{2}(\tau)$-manifolds [5], [4] (cf. [2, Ch. IX, Theorem 7.3]): any two $\ell_{2}(\tau)$-manifolds with the same homotopy type are homeomorphic.

COROLlary 1 (Classification). Two open subsets of $F$ are homeomorphic if they have the same homotopy type.

Due to the stability theorem for $\ell_{2}(\tau)$-manifolds [9] (cf. [2, Ch. IX, Theorem 4.1]), $M \times \ell_{2}(\tau) \approx M$ for every $\ell_{2}(\tau)$-manifold $M$, hence we have the following:

Corollary 2 (Stability). Every open set $U$ in $F$ is homeomorphic to $U \times F$.

For each connected $\ell_{2}(\tau)$-manifold $M$, there exists a locally finite-dimensional simplicial complex $K$ with card $K^{(0)} \leq \tau$ such that $M \approx|K| \times \ell_{2}$, where $|K|$ admits the metric topology, by the triangulation theorem for $\ell_{2}(\tau)$ manifolds [4]. Thus, the following holds:

Corollary 3 (Triangulation). Each open subset of $F$ is homeomorphic to $|K| \times F$ for some locally finite-dimensional simplicial complex $K$ with card $K^{(0)} \leq \tau$. 
2. Outline of the proof. Let $\mathbf{I}=[0,1]$ and $\mathbb{R}_{+}=[0, \infty)$. Since $\ell_{2}(\tau) \times$ $\mathbb{R}_{+}^{n} \approx \ell_{2}(\tau) \times \mathbf{I}^{n} \approx \ell_{2}(\tau)$ for every $n \in \mathbb{N}$ (cf. [10]), it follows from the stability theorem for $\ell_{2}(\tau)$-manifolds [9] that $X \times \mathbb{R}_{+}^{n} \approx X \times \mathbf{I}^{n} \approx X$ for each $\ell_{2}(\tau)$-manifold $X$ and $n \in \mathbb{N}$. Moreover, $\mathbb{R}^{\infty} \approx \mathbb{R}_{+}^{\infty}=\underline{\lim } \mathbb{R}_{+}^{n}$, the direct limit of the tower $\mathbb{R}_{+} \subset \mathbb{R}_{+}^{2} \subset \mathbb{R}_{+}^{3} \subset \cdots$ (cf. [7]), where each $\mathbb{R}_{+}^{n}$ is identified with $\mathbb{R}_{+}^{n} \times\{0\} \subset \mathbb{R}_{+}^{n+1}$. Therefore, $F \approx \ell_{2}(\tau) \times \mathbb{R}_{+}^{\infty}$. Thus, we can consider $U$ as an open set in $\ell_{2}(\tau) \times \mathbb{R}_{+}^{\infty}$. One should note that

$$
\ell_{2}(\tau) \times \mathbb{R}_{+}^{\infty}=\ell_{2}(\tau) \times \lim _{\longrightarrow} \mathbb{R}_{+}^{n} \neq \lim _{\longrightarrow}\left(\ell_{2}(\tau) \times \mathbb{R}_{+}^{n}\right) \text { as spaces. }
$$

A closed set $A$ in a space $X$ is called a $Z$-set if for each open cover $\mathcal{U}$ of $X$ there is a map $f: X \rightarrow X \backslash A$ which is $\mathcal{U}$-close to id, that is, every $\{x, f(x)\}$ is contained in some $U \in \mathcal{U}$. It is known that if an $\ell_{2}(\tau)$-manifold $A$ is a $Z$-set in an $\ell_{2}(\tau)$-manifold $X$ then $A$ is collared in $X$, that is, there is an open embedding $\psi: A \times[0,1) \rightarrow X$ (called a collar) such that $\psi(x, 0)=x$ for every $x \in A$.

For each $n \in \mathbb{N}$, let $U_{n}=U \cap\left(\ell_{2}(\tau) \times \mathbb{R}_{+}^{n}\right)$. As is easily observed, each $U_{n}$ is an $\ell_{2}(\tau)$-manifold which is a $Z$-set in $U_{n+1}$. Note that $U_{1} \subset U_{2} \subset \ldots$ and $U=\bigcup_{n \in \mathbb{N}} U_{n}$. We define

$$
M=\bigcup_{n \in \mathbb{N}}[n-1, n] \times U_{n} \subset \bigcup_{n \in \mathbb{N}} \mathbb{R}_{+} \times \ell_{2}(\tau) \times \mathbb{R}_{+}^{n}=\mathbb{R}_{+} \times \ell_{2}(\tau) \times \mathbb{R}_{+}^{\infty} .
$$

Now, each $[n-1, n] \times U_{n}$ is an $\ell_{2}(\tau)$-manifold and

$$
\left([n-1, n] \times U_{n}\right) \cap\left([n, n+1] \times U_{n+1}\right)=\{n\} \times U_{n},
$$

where $\{n\} \times U_{n}$ is collared not only in $[n-1, n] \times U_{n}$ but also in $[n, n+1] \times U_{n+1}$ because it is a $Z$-set in the $\ell_{2}(\tau)$-manifold $[n, n+1] \times U_{n+1}$. It follows that $M$ is a separable $\ell_{2}(\tau)$-manifold. Since $\mathbb{R}^{\infty} \approx[0,1)^{\infty}=\lim _{\longrightarrow}[0,1)^{n}$, we shall show that $M \times[0,1)^{\infty} \approx U$.

Let $\Psi=\left(\psi_{i}\right)_{i \in \mathbb{N}}$ be a sequence of collars $\psi_{i}: U_{i} \times[0,1) \rightarrow U_{i+1}$. By the natural embedding

$$
\psi_{n} \times \text { id }: U_{n} \times[0,1) \times[0,1)^{\infty} \rightarrow U_{n+1} \times[0,1)^{\infty},
$$

we regard $U_{n} \times[0,1) \times[0,1)^{\infty}=U_{n} \times[0,1)^{\infty}$ as an open set in $U_{n+1} \times[0,1)^{\infty}$. Let $U_{\Psi}$ be the direct limit of the following open tower:

$$
U_{1} \times[0,1)^{\infty} \underset{\psi_{1} \times \text { id }}{\subset} U_{2} \times[0,1)^{\infty} \underset{\psi_{2} \times \text { id }}{\subset} \cdots .
$$

Since each $U_{n} \times[0,1)^{\infty}$ is an open set in $\ell_{2}(\tau) \times \mathbb{R}_{+}^{n} \times[0,1)^{\infty} \approx \ell_{2}(\tau) \times \mathbb{R}^{\infty}$, it follows that $U_{\Psi}$ is an $\ell_{2}(\tau) \times \mathbb{R}^{\infty}$-manifold. Since $U_{n} \times[0,1)^{k} \subset U_{n+k}$ for each $n, k \in \mathbb{N}$, we can regard $U_{\Psi}=\bigcup_{n \in \mathbb{N}} U_{n}$ as sets but the topology of $U_{\Psi}$ depends on the sequence $\Psi=\left(\psi_{i}\right)_{i \in \mathbb{N}}$. The first step of the proof is to find $\Psi$ so that $U_{\Psi} \approx U$. 
Observe that $U_{\Psi}$ is also the direct limit of the following open tower:

$$
U_{1} \times[0,1 / 2)^{\infty} \underset{\psi_{1} \times \mathrm{id}}{\subset} U_{2} \times[0,2 / 3)^{\infty} \underset{\psi_{2} \times \mathrm{id}}{\subset} \cdots
$$

On the other hand, for each $n \in \mathbb{N}$, let

$$
M_{n}^{\infty}=\left(\bigcup_{i=1}^{n}[i-1, n) \times U_{i}\right) \times\left[0, \frac{n}{n+1}\right)^{\infty} .
$$

Then $M_{1}^{\infty} \subset M_{2}^{\infty} \subset \cdots$ are open sets in $M \times[0,1)^{\infty}$ and $M \times[0,1)^{\infty}=$ $\bigcup_{n \in \mathbb{N}} M_{n}^{\infty}$. In the second step, we construct homeomorphisms

$$
h_{n}: M_{n}^{\infty} \rightarrow U_{n} \times\left[0, \frac{n}{n+1}\right)^{\infty}, \quad n \in \mathbb{N},
$$

so that the following diagram commutes:

$$
\begin{array}{ccc}
M_{n}^{\infty} & \multicolumn{1}{c}{M_{n+1}^{\infty}} \\
h_{n} \downarrow & \downarrow h_{n+1} \\
U_{n} \times\left[0, \frac{n}{n+1}\right)^{\infty} \underset{\psi_{n} \times \mathrm{id}}{\stackrel{\subset}{\longrightarrow}} U_{n+1} \times\left[0, \frac{n+1}{n+2}\right)^{\infty}
\end{array}
$$

This implies that $M \times[0,1)^{\infty} \approx U_{\Psi}$.

To complete the proof, we use two more results on $\ell_{2}(\tau)$-manifolds. The following is proved in [5]:

THEOREM 1. Let $M$ and $N$ be $\ell_{2}(\tau)$-manifolds. Every homotopy equivalence $f: M \rightarrow N$ is homotopic to $(\simeq)$ a homeomorphism.

We call an embedding $f: X \rightarrow Y$ a $Z$-embedding if $f(X)$ is a $Z$-set in $Y$. The following easily follows from the $Z$-set unknotting theorem [1]:

Theorem 2. Let $f: M \rightarrow N$ be a homeomorphism between $\ell_{2}(\tau)$ manifolds and $g: A \rightarrow N$ a $Z$-embedding of a $Z$-set $A$ in $M$. If $g$ is homotopic to the restriction $f \mid A$ then $g$ extends to a homeomorphism $\tilde{g}: M \rightarrow M$ which is isotopic to $f$.

3. The first step of the proof. For simplicity, we use the following notation:

$$
\prod_{i=k}^{n<\omega}\left[0, a_{i}\right]=\bigcup_{n \geq k} \prod_{i=k}^{n}\left[0, a_{i}\right] \quad \text { for } a_{i}>0, i \geq k .
$$

For a subset $N \subset \ell_{2}(\tau) \times \mathbb{R}_{+}^{n}$ and a map $\alpha: N \rightarrow(0,1)$, we define

$$
N(\alpha)=\left\{(x, t) \in N \times \mathbb{R}_{+} \mid t<\alpha(x)\right\} \subset \ell_{2}(\tau) \times \mathbb{R}_{+}^{n+1} .
$$

For each $n \in \mathbb{N}$, let $U_{n}=U \cap\left(\ell_{2}(\tau) \times \mathbb{R}_{+}^{n}\right)$. Then $U_{n}$ is an $\ell_{2}(\tau)$-manifold. For a sequence $\alpha=\left(\alpha_{k}\right)_{k \in \mathbb{N}}$ of maps $\alpha_{k}: U_{k} \rightarrow(0,1)$ satisfying the condition 
$U_{k}\left(\alpha_{k}\right) \subset U_{k+1}$, we can inductively define

$$
\begin{aligned}
U_{n}\left(\alpha_{n}, \ldots, \alpha_{k}\right) & =U_{n}\left(\alpha_{n}, \ldots, \alpha_{k-1}\right)\left(\alpha_{k}\right) \\
& \subset U_{k}\left(\alpha_{k}\right) \subset U_{k+1} \quad \text { for each } k>n .
\end{aligned}
$$

Then, for each $n \in \mathbb{N}$,

$$
U_{n}\left(\alpha_{n}\right) \subset U_{n}\left(\alpha_{n}, \alpha_{n+1}\right) \subset U_{n}\left(\alpha_{n}, \alpha_{n+1}, \alpha_{n+2}\right) \subset \cdots .
$$

Let $U_{n}^{\alpha}=\bigcup_{k \geq n} U_{n}\left(\alpha_{n}, \ldots, \alpha_{k}\right) \subset U$. Thus, we have a tower $U_{1}^{\alpha} \subset U_{2}^{\alpha} \subset$ $U_{3}^{\alpha} \subset \cdots$ with $U=\bigcup_{n \in \mathbb{N}} U_{n}^{\alpha}$. If each $U_{n}^{\alpha}$ is open in $U$ then $U=\lim _{n} U_{n}^{\alpha}$.

Lemma 1. There exists a sequence $\alpha=\left(\alpha_{k}\right)_{k \in \mathbb{N}}$ of maps $\alpha_{k}: U_{k} \rightarrow(0,1)$ such that $U_{k}\left(\alpha_{k}\right) \subset U_{k+1}$ for every $k \in \mathbb{N}$ and each $U_{n}^{\alpha}$ is open in $U$, hence $U=\lim U_{n}^{\alpha}$. Moreover, for each $x \in U_{k}$ there is a neighborhood $V$ of $x$ in $U_{k}$ and $a_{i}>0, i>k$, such that $\inf _{y \in V} \alpha_{k}(y)>0$ and

$$
\inf \left\{\alpha_{n}(y) \mid y \in V \times \prod_{i=k+1}^{n}\left[0, a_{i}\right]\right\}>0 \quad \text { for every } n>k .
$$

Proof. For each $k \in \mathbb{N}$, let $\mathcal{V}_{k}$ be a locally finite open cover of $U_{k}$ and let $a_{V, i} \in(0,1], i>k$, be such that

$$
\operatorname{cl} V \times \prod_{i=k+1}^{n<\omega}\left[0, a_{V, i}\right] \subset U \quad \text { for each } k \in \mathbb{N} \text { and } V \in \mathcal{V}_{k} .
$$

We define $\beta_{k}: U_{k} \rightarrow \mathbf{I}$ as follows:

$$
\beta_{k}(x)=\max \left\{a_{V, k+1} \mid V \in \mathcal{V}_{j}, j \leq k, x \in \operatorname{cl} V \times \prod_{i=j+1}^{k}\left[0, a_{V, i}\right]\right\},
$$

where $\operatorname{cl} V \times \prod_{i=j+1}^{k}\left[0, a_{V, i}\right]=\operatorname{cl} V$ if $j=k$. Then $\beta_{k}$ is upper semicontinuous because

$$
\left\{(x, t) \in U_{k} \times \mathbf{I} \mid t \leq \beta_{k}(x)\right\}=\bigcup_{j \leq k} \bigcup_{V \in \mathcal{V}_{j}} \operatorname{cl} V \times \prod_{i=j+1}^{k+1}\left[0, a_{V, i}\right]
$$

is closed in $U_{k} \times \mathbb{R}_{+}$. Choose an open set $U_{k+1}^{\prime}$ in $U_{k+1}$ so that

$$
\left\{(x, t) \in U_{k} \times \mathbf{I} \mid t \leq \beta_{k+1}(x)\right\} \subset U_{k+1}^{\prime} \subset \operatorname{cl} U_{k+1}^{\prime} \subset U_{k+1} .
$$

Then we have a lower semicontinuous function $\gamma_{k}: U_{k} \rightarrow \mathbf{I}$ defined by

$$
\gamma_{k}(x)=\sup \left\{t \in \mathbf{I} \mid\{x\} \times[0, t] \subset U_{k+1}^{\prime}\right\} .
$$

Since $\beta_{k}<\gamma_{k}$, there exists a continuous map $\alpha_{k}: U_{k} \rightarrow(0,1)$ such that $\beta_{k}<\alpha_{k}<\gamma_{k}$. Thus, $U_{k}\left(\alpha_{k}\right) \subset U_{k+1}$ for every $k \in \mathbb{N}$.

By the definition, for each $V \in \mathcal{V}_{k}$ and $n \geq k$,

$$
\operatorname{cl} V \times \prod_{i=k+1}^{n+1}\left[0, a_{V, i}\right] \subset U_{k}\left(\alpha_{k}, \ldots, \alpha_{n}\right),
$$


which implies $\inf _{y \in V} \alpha_{k}(y) \geq a_{V, k+1}>0$ and

$$
\inf \left\{\alpha_{n}(y) \mid y \in V \times \prod_{i=k+1}^{n}\left[0, a_{i}\right]\right\} \geq a_{V, n+1}>0 \quad \text { for every } n>k .
$$

To show that each $U_{n}^{\alpha}$ is open in $U$, let $x \in U_{n}^{\alpha}$. Choose $k \geq n$ so that $x \in U_{n}\left(\alpha_{n}, \ldots, \alpha_{k}\right) \subset U_{k+1}$. Then $x$ has the following open neighborhood in $U$ :

$$
W \times \prod_{i=k+2}^{m+1<\omega}\left[0, a_{V, i}\right)
$$

where $W=V \cap U_{n}\left(\alpha_{n}, \ldots, \alpha_{k}\right)$ and $V \in \mathcal{V}_{k+1}$. Now, by induction on $m>k$, we shall show that

$$
W \times \prod_{i=k+2}^{m+1}\left[0, a_{V, i}\right] \subset U_{n}\left(\alpha_{n}, \ldots, \alpha_{m}\right) .
$$

To this end, take an arbitrary

$$
y=\left(z, t_{k+2}, \ldots, t_{m+1}\right) \in W \times \prod_{i=k+2}^{m+1}\left[0, a_{V, i}\right] .
$$

By the inductive assumption, it follows that

$$
y^{\prime}=\left(z, t_{k+2}, \ldots, t_{m}\right) \in W \times \prod_{i=k+2}^{m}\left[0, a_{V, i}\right] \subset U_{n}\left(\alpha_{n}, \ldots, \alpha_{m-1}\right) .
$$

Since $t_{m+1}<a_{V, m+1}<\alpha_{m}\left(y^{\prime}\right)$, it follows that

$$
y \in U_{n}\left(\alpha_{n}, \ldots, \alpha_{m-1}\right)\left(\alpha_{m}\right)=U_{n}\left(\alpha_{n}, \ldots, \alpha_{m}\right) .
$$

Thus, we have

$$
W \times \prod_{i=k+2}^{m+1<\omega}\left[0, a_{V, i}\right]=\bigcup_{m>k} W \times \prod_{i=k+2}^{m}\left[0, a_{V, i}\right] \subset U_{n}^{\alpha} .
$$

Therefore, $U_{n}^{\alpha}$ is open in $U$.

Now, we shall construct a sequence $\Psi=\left(\psi_{i}\right)_{i \in \mathbb{N}}$ of collars $\psi_{i}: U_{i} \times[0,1) \rightarrow$ $U_{i+1}, i \in \mathbb{N}$, so that $U_{\Psi} \approx U$. Recall $U_{\Psi}$ is the direct limit of the following open tower:

$$
U_{1} \times[0,1)^{\infty} \underset{\psi_{1} \times \mathrm{id}}{\subset} U_{2} \times[0,1)^{\infty} \underset{\psi_{2} \times \mathrm{id}}{\subset} \cdots,
$$

where we regard $U_{n} \times[0,1)^{\infty}$ as an open set in $U_{n+1} \times[0,1)^{\infty}$ by the embedding

$$
\psi_{n} \times \mathrm{id}: U_{n} \times[0,1)^{\infty}=U_{n} \times[0,1) \times[0,1)^{\infty} \rightarrow U_{n+1} \times[0,1)^{\infty} .
$$

LEMma 2. There exists a sequence $\Psi=\left(\psi_{n}\right)_{n \in \mathbb{N}}$ of collars $\psi_{n}: U_{n} \times$ $[0,1) \rightarrow U_{n+1}$ such that $U_{\Psi} \approx U$. 
Proof. Let $\alpha=\left(\alpha_{n}\right)_{n \in \mathbb{N}}$ be a sequence of maps $\alpha_{n}: U_{n} \rightarrow(0,1)$ obtained by Lemma 1. Then $U_{n}^{\alpha}$ is open in $U$ and $U=\lim U_{n}^{\alpha}$. For each $n \in \mathbb{N}$, we define a collar $\psi_{n}: U_{n} \times[0,1) \rightarrow U_{n+1}$ by $\psi_{n}(x, t)=\left(x, \alpha_{n}(x) t\right)$. For every $k \in \mathbb{N}$, we inductively define $\delta_{n, k}: U_{n} \times[0,1)^{k} \rightarrow[0,1)$ as follows:

$$
\begin{aligned}
& \delta_{n, k}\left(x, t_{n+1}, \ldots, t_{n+k}\right) \\
& \quad=\alpha_{n+k-1}\left(x, \delta_{n, 1}\left(x, t_{n+1}\right), \ldots, \delta_{n, k-1}\left(x, t_{n+1}, \ldots, t_{n+k-1}\right)\right) t_{n+k},
\end{aligned}
$$

where $\delta_{n, 1}(x, t)=\alpha_{n}(x) t$. Then we have the following equation:

$$
\delta_{n, k}\left(x, \frac{s_{n+1}}{\alpha_{n}(x)}, \ldots, \frac{s_{n+k}}{\alpha_{n+k-1}\left(x, s_{n+1}, \ldots, s_{n+k-1}\right)}\right)=s_{n+k} .
$$

Define $h_{n}: U_{n} \times[0,1)^{\infty} \rightarrow U_{n}^{\alpha}$ and $g_{n}: U_{n}^{\alpha} \rightarrow U_{n} \times[0,1)^{\infty}$ as follows:

$h_{n}\left(x, t_{n+1}, t_{n+2}, \ldots\right)=\left(x, \delta_{n, 1}\left(x, t_{n+1}\right), \delta_{n, 2}\left(x, t_{n+1}, t_{n+2}\right), \ldots\right)$,

$g_{n}\left(x, s_{n+1}, s_{n+2}, \ldots\right)=\left(x, \frac{s_{n+1}}{\alpha_{n}(x)}, \frac{s_{n+2}}{\alpha_{n+1}\left(x, s_{n+1}\right)}, \frac{s_{n+3}}{\alpha_{n+2}\left(x, s_{n+1}, s_{n+2}\right)}, \ldots\right)$.

It is easily observed that $g_{n} \circ h_{n}=\mathrm{id}_{U_{n} \times[0,1)^{\infty}}$. By $(*)$, we have $h_{n} \circ g_{n}=\mathrm{id}_{U_{n}^{\alpha}}$. Thus, $g_{n}$ is a bijection with $h_{n}=g_{n}^{-1}$. Moreover, $\left(\psi_{n} \times\right.$ id $) \circ g_{n}=g_{n+1} \mid U_{n}^{\alpha}$ for all $n \in \mathbb{N}$, that is, the following diagram commutes:

$$
\begin{array}{ccc}
U_{n}^{\alpha} & \subset & U_{n+1}^{\alpha} \\
g_{n} \downarrow & & g_{n+1} \downarrow \\
U_{n} \times[0,1)^{\infty} & \underset{\psi_{n} \times \mathrm{id}}{\stackrel{\subset}{\longrightarrow}} & U_{n+1} \times[0,1)^{\infty}
\end{array}
$$

Indeed, for each $\left(x, s_{n+1}, s_{n+2}, \ldots\right) \in U_{n}^{\alpha}$,

$$
\begin{aligned}
\left(\psi_{n} \times \mathrm{id}\right) \circ g_{n}\left(x, s_{n+1}, s_{n+2}, \ldots\right) & =\left(\psi_{n} \times \mathrm{id}\right)\left(x, \frac{s_{n+1}}{\alpha_{n}(x)}, \frac{s_{n+2}}{\alpha_{n+1}\left(x, s_{n+1}\right)}, \ldots\right) \\
& =\left(\psi_{n}\left(x, \frac{s_{n+1}}{\alpha_{n}(x)}\right), \frac{s_{n+2}}{\alpha_{n+1}\left(x, s_{n+1}\right)}, \ldots\right) \\
& =\left(\left(x, s_{n+1}\right), \frac{s_{n+2}}{\alpha_{n+1}\left(x, s_{n+1}\right)}, \ldots\right) \\
& =g_{n+1}\left(\left(x, s_{n+1}\right), s_{n+2}, \ldots\right) .
\end{aligned}
$$

We shall show that $h_{n}$ and $g_{n}$ are all continuous, which means that $g_{n}$ is a homeomorphism. Then we shall have

$$
U=\lim _{\longrightarrow} U_{n}^{\alpha} \approx \lim _{\longrightarrow} U_{n} \times[0,1)^{\infty}=U_{\Psi} .
$$

To see the continuity of $h_{n}$ at $x \in U_{n} \times[0,1)^{\infty}$, let $V$ be a neighborhood of $h_{n}(x)$ in $U_{n}^{\alpha}$. Then $x$ is contained in some $U_{n} \times[0,1)^{k}$, which implies that $h_{n}(x) \in U_{n}\left(\alpha_{1}, \ldots, \alpha_{k-1}\right)$. We can find a neighborhood $V^{\prime}$ of $h_{n}(x)$ in 
$U_{n} \times[0,1)^{k}$ and $0<r_{n+k+i}<1, i \in \mathbb{N}$, such that

$$
h_{n}(x) \in V^{\prime} \times \prod_{i=1}^{j<\omega}\left[0, r_{n+k+i}\right] \subset V .
$$

Since $\delta_{n, 1}, \ldots, \delta_{n, k}$ are continuous, it follows that $h_{n} \mid U_{n} \times[0,1)^{k}$ is continuous, hence $x$ has a neighborhood $W$ in $U_{n} \times[0,1)^{k}$ such that $h_{n}(W) \subset V^{\prime}$. Then $W \times \prod_{i=1}^{j<\omega}\left[0, r_{n+k+i}\right]$ is a neighborhood of $x$ in $U_{n} \times[0,1)^{\infty}$ and

$$
h_{n}\left(W \times \prod_{i=1}^{j<\omega}\left[0, r_{n+k+i}\right]\right) \subset V^{\prime} \times \prod_{i=1}^{j<\omega}\left[0, r_{n+k+i}\right] \subset V,
$$

which implies that $h_{n}$ is continuous at $x$.

To see the continuity of $g_{n}$ at $x \in U_{n}^{\alpha}$, for each neighborhood $V$ of $g_{n}(x)$ in $U_{n} \times[0,1)^{\infty}$ choose an open set $W$ in $U_{n}$ and $r_{n+i}>0, i \in \mathbb{N}$, so that

$$
g_{n}(x) \in W \times \prod_{i=1}^{j<\omega}\left[0, r_{n+i}\right] \subset V .
$$

Due to Lemma 1, it can be assumed that $\inf _{y \in W} \alpha_{n}(y)>0$ and

$$
\inf \left\{\alpha_{n+k}(y) \mid y \in W \times \prod_{i=1}^{k}\left[0, r_{n+i}\right]\right\}>0 \quad \text { for every } k \in \mathbb{N} .
$$

Hence, we can find $0<q_{n+i} \leq r_{n+i}, i \in \mathbb{N}$, such that

$$
\begin{aligned}
\left(y, s_{n+1}, \ldots, s_{n+j-1}\right) \in W \times & \prod_{i=1}^{j}\left[0, r_{n+i}\right], \quad s_{n+j}<q_{n+j}, \\
& \Rightarrow \frac{s_{n+j}}{\alpha_{n+j-1}\left(y, s_{n+1}, \ldots, s_{n+j-1}\right)}<r_{n+j} .
\end{aligned}
$$

Then it follows that

$$
g_{n}\left(W \times \prod_{i=1}^{j<\omega}\left[0, q_{n+i}\right]\right) \subset W \times \prod_{i=1}^{j<\omega}\left[0, r_{n+i}\right] \subset V,
$$

which implies that $g_{n}$ is continuous at $x$.

REMARK 1. Let $M_{1} \subset M_{2} \subset \cdots$ be a closed tower of $\ell_{2}(\tau)$-manifolds such that each $M_{i}$ is a $Z$-set (hence collared) in $M_{i+1}$. Then $M_{\infty}=\bigcup_{i \in \mathbb{N}} M_{n}$ has a topology such that $M_{\infty}$ is an $\ell_{2}(\tau) \times \mathbb{R}^{\infty}$-manifold and each $M_{i}$ is a subspace of $M_{\infty}$. Indeed, given a sequence $\Psi=\left(\psi_{i}\right)_{i \in \mathbb{N}}$ of collars $\psi_{i}$ : $M_{i} \times[0,1) \rightarrow M_{i+1}$, we regard $M_{n} \times[0,1)^{\infty}$ as an open set in $M_{n+1} \times[0,1)^{\infty}$ by the natural embedding

$$
\psi_{n} \times \mathrm{id}: M_{n} \times[0,1)^{\infty}=M_{n} \times[0,1) \times[0,1)^{\infty} \rightarrow M_{n+1} \times[0,1)^{\infty} .
$$


Let $M_{\Psi}$ be the direct limit of the open tower

$$
M_{1} \times[0,1)^{\infty} \underset{\psi_{1} \times \text { id }}{\subset} M_{2} \times[0,1)^{\infty} \underset{\psi_{2} \times \text { id }}{\subset} \cdots .
$$

Since every separable $\ell_{2}(\tau)$-manifold can be embedded into $\ell_{2}(\tau)$ as an open set by the open embedding theorem for $\ell_{2}(\tau)$-manifolds [5] (cf. [4]), each $M_{n} \times[0,1)^{\infty}$ is homeomorphic to an open set in $\ell_{2}(\tau) \times[0,1)^{\infty} \approx \ell_{2}(\tau) \times \mathbb{R}^{\infty}$. Then $M_{\Psi}$ is an $\ell_{2}(\tau) \times \mathbb{R}^{\infty}$-manifold. Since $M_{n} \times[0,1)^{k} \subset M_{n+k}$ for each $n, k \in \mathbb{N}$, we can regard $M_{\Psi}=M_{\infty}$ as sets but the topology of $M_{\Psi}$ depends on the sequence $\Psi=\left(\psi_{i}\right)_{i \in \mathbb{N}}$. One should note that $M_{\Psi} \neq \lim _{\longrightarrow} M_{n}$. In fact, the topology of $\lim _{\longrightarrow} M_{n}$ is finer than that of $M_{\Psi}$.

4. The second step of the proof. By Lemma 2, we have a sequence $\Psi=\left(\psi_{i}\right)_{i \in \mathbb{N}}$ of collars $\psi_{i}: U_{i} \times \mathbf{I} \rightarrow U_{i+1}$ such that $U$ is homeomorphic to the direct limit $U_{\Psi}$ of the following open tower:

$$
U_{1} \times[0,1)^{\infty} \underset{\psi_{1} \times \mathrm{id}}{\subset} U_{2} \times[0,1)^{\infty} \underset{\psi_{2} \times \mathrm{id}}{\subset} \cdots
$$

The Main Theorem is reduced to the following:

Lemma 3. $M \times[0,1)^{\infty} \approx U_{\Psi}$.

Proof. Here, we regard $U_{\Psi}$ as the direct limit of the following open tower:

$$
U_{1} \times[0,1 / 2)^{\infty} \underset{\psi_{1} \times \mathrm{id}}{\subset} U_{2} \times[0,2 / 3)^{\infty} \underset{\psi_{2} \times \mathrm{id}}{\subset} \cdots .
$$

Recall we can write $M \times[0,1)^{\infty}=\bigcup_{n \in \mathbb{N}} M_{n}^{\infty}$, where $M_{1}^{\infty} \subset M_{2}^{\infty} \subset \cdots$ are open sets in $M \times[0,1)^{\infty}$ defined as follows:

$$
M_{n}^{\infty}=\left(\bigcup_{i=1}^{n}[i-1, n) \times U_{i}\right) \times\left[0, \frac{n}{n+1}\right)^{\infty} .
$$

To show that $M \times[0,1)^{\infty} \approx U_{\Psi}$, it suffices construct homeomorphisms

$$
h_{n}: M_{n}^{\infty} \rightarrow U_{n} \times\left[0, \frac{n}{n+1}\right)^{\infty}, \quad n \in \mathbb{N},
$$

so that the following diagram commutes:

$$
\begin{array}{ccc}
M_{n}^{\infty} & \subset & M_{n+1}^{\infty} \\
h_{n} \downarrow & & \downarrow^{h_{n+1}} \\
U_{n} \times\left[0, \frac{n}{n+1}\right)^{\infty} \underset{\psi_{n} \times \mathrm{id}}{\stackrel{\subset}{\longrightarrow}} U_{n+1} \times\left[0, \frac{n+1}{n+2}\right)^{\infty}
\end{array}
$$

For each $n \in \mathbb{N}$, we define

$$
M_{n}=\left(\bigcup_{i=1}^{n}[i-1, n) \times U_{i}\right) \times\left[0, \frac{n}{n+1}\right)^{n} .
$$


Then it follows that

$$
M_{n}^{\infty}=M_{n} \times\left[0, \frac{n}{n+1}\right)^{\infty} \text { and } M_{n} \times\left[0, \frac{n}{n+1}\right) \subset M_{n+1} .
$$

If we could construct homeomorphisms

$$
f_{n}: M_{n} \rightarrow U_{n} \times\left[0, \frac{n}{n+1}\right)^{\infty}, \quad n \in \mathbb{N},
$$

so that the following diagram commutes:

$$
\begin{array}{ccc}
M_{n} \times\left[0, \frac{n}{n+1}\right) & \stackrel{c}{\longrightarrow} & M_{n+1} \\
f_{n} \times \text { id } & & \\
U_{n} \times\left[0, \frac{n}{n+1}\right)^{2} \underset{\psi \times \text { id }}{\longrightarrow} & U_{n+1} \times\left[0, \frac{n+1}{n+2}\right)
\end{array}
$$

then the desired homeomorphism $h_{n}$ could be defined as follows:

$$
h_{n}=f_{n} \times \text { id }: M_{n}^{\infty}=M_{n} \times\left[0, \frac{n}{n+1}\right)^{\infty} \rightarrow U_{n} \times\left[0, \frac{n}{n+1}\right)^{\infty} .
$$

To construct $f_{n}$ inductively, let

$$
\begin{aligned}
\bar{M}_{n}= & \left(\bigcup_{i=1}^{n}[i-1, n] \times U_{i}\right) \times\left[0, \frac{n}{n+1}\right]^{n}, \\
\partial M_{n}= & \bar{M}_{n} \backslash M_{n} \\
= & \{n\} \times U_{n} \times\left[0, \frac{n}{n+1}\right]^{n} \\
& \cup\left(\bigcup_{i=1}^{n}[i-1, n] \times U_{i}\right) \times\left(\left[0, \frac{n}{n+1}\right]^{n} \backslash\left[0, \frac{n}{n+1}\right)^{n}\right) .
\end{aligned}
$$

Similarly to $M$, we can see that these are $\ell_{2}(\tau)$-manifolds. Note that $\partial M_{n}$ is a $Z$-set in $\bar{M}_{n}$. Let $p_{n}: \bar{M}_{n} \rightarrow U_{n}$ be the projection and $i_{n}: U_{n} \rightarrow \partial M_{n} \subset \bar{M}_{n}$ the injection defined by $i_{n}(x)=\left(n, x, v_{n}\right)$, where

$$
v_{n}=\left(\frac{n}{n+1}, \ldots, \frac{n}{n+1}\right) \in\left[0, \frac{n}{n+1}\right]^{n} .
$$

Then $i_{n}\left(U_{n}\right)=U_{n} \times\left\{v_{n}\right\}$ is a strong deformation retract of both $\bar{M}_{n}$ and $\partial M_{n}$, hence $p_{n}$ and $p_{n} \mid \partial M_{n}$ are homotopy equivalences and $i_{n}$ is a homotopy inverse of both $p_{n}$ and $p_{n} \mid \partial M_{n}$. Thus, we have the homotopy equivalences

$$
r_{n}: \bar{M}_{n} \rightarrow U_{n} \times\left[0, \frac{n}{n+1}\right] \quad \text { and } \quad r_{n}^{\prime}=r_{n} \mid \partial M_{n}: \partial M_{n} \rightarrow U_{n} \times\left\{\frac{n}{n+1}\right\}
$$

defined by $r_{n}(x)=\left(p_{n}(x), n /(n+1)\right)$. 
We shall construct homeomorphisms

$$
\bar{f}_{n}: \bar{M}_{n} \rightarrow U_{n} \times\left[0, \frac{n}{n+1}\right], \quad n \in \mathbb{N},
$$

so that $\bar{f}_{n} \simeq r_{n}$,

$$
\bar{f}_{n}\left(\partial M_{n}\right)=U_{n} \times\left\{\frac{n}{n+1}\right\}, \quad \text { i.e., } \quad \bar{f}_{n}\left(M_{n}\right)=U_{n} \times\left[0, \frac{n}{n+1}\right),
$$

and the following diagram commutes:

$$
\begin{array}{ccc}
\bar{M}_{n} \times\left[0, \frac{n}{n+1}\right] & \stackrel{M_{n+1}}{\longrightarrow} & \bar{f}_{n+1} \\
\bar{f}_{n} \times \text { id } & \\
U_{n} \times\left[0, \frac{n}{n+1}\right]^{2} \underset{\psi_{n} \times \text { id }}{\longrightarrow} & U_{n+1} \times\left[0, \frac{n+1}{n+2}\right]
\end{array}
$$

Then $f_{n}=\bar{f}_{n} \mid M_{n}$ is the desired homeomorphism.

First, by Theorem 1, we have homeomorphisms $f: \bar{M}_{1} \rightarrow U_{1} \times[0,1 / 2]$ and $f^{\prime}: \partial M_{1} \rightarrow U_{1} \times\{1 / 2\}$ onto $U_{1}$ such that $f \simeq r_{1}$ and $f^{\prime} \simeq r_{1}^{\prime}$. Since $f^{\prime} \simeq f \mid \partial M_{1}$, we can apply Theorem 2 to extend $f^{\prime}$ to a homeomorphism $\bar{f}_{1}: \bar{M}_{1} \rightarrow U_{1} \times[0,1 / 2]$ which is isotopic $f$, hence $\bar{f}_{1} \simeq r_{1}$.

Now, assume that $\bar{f}_{n}$ has been obtained and consider the following sets:

$$
\begin{aligned}
\bar{\partial} M_{n}= & \partial M_{n} \times\left[0, \frac{n}{n+1}\right] \cup \bar{M}_{n} \times\left\{\frac{n}{n+1}\right\}, \\
L_{n+1}= & \bar{M}_{n+1} \backslash\left(M_{n} \times\left[0, \frac{n}{n+1}\right)\right) \\
= & {[n, n+1] \times U_{n+1} \times\left[0, \frac{n+1}{n+2}\right]^{n+1} } \\
& \cup\left(\bigcup_{i=1}^{n}[i-1, n] \times U_{i}\right) \times\left(\left[0, \frac{n+1}{n+2}\right]^{n+1} \backslash\left[0, \frac{n}{n+1}\right)^{n+1}\right), \\
B_{n}= & \psi_{n}\left(U_{n} \times\left\{\frac{n}{n+1}\right\}\right) \times\left[0, \frac{n}{n+1}\right] \\
& \cup \psi_{n}\left(U_{n} \times\left[0, \frac{n}{n+1}\right]\right) \times\left\{\frac{n}{n+1}\right\}, \\
W_{n+1}= & \left(U_{n+1} \times\left[0, \frac{n+1}{n+2}\right]\right) \backslash\left(\psi_{n}\left(U_{n} \times\left[0, \frac{n}{n+1}\right)\right) \times\left[0, \frac{n}{n+1}\right)\right) .
\end{aligned}
$$

Then we have the following homeomorphism:

$$
g_{n}=\left(\psi_{n} \times \mathrm{id}\right)\left(\bar{f}_{n} \times \mathrm{id}\right) \mid \bar{\partial} M_{n}: \bar{\partial} M_{n} \rightarrow B_{n} .
$$

Observe that $L_{n+1}$ and $W_{n+1}$ are $\ell_{2}(\tau)$-manifolds, $\bar{\partial} M_{n}$ and $\partial M_{n+1}$ are disjoint $Z$-sets in $L_{n+1}$, and $B_{n}$ and $U_{n+1} \times\{(n+1) /(n+2)\}$ are disjoint $Z$ - 
sets in $W_{n+1}$. Since $i_{n+1}\left(U_{n+1}\right)=U_{n+1} \times\left\{v_{n+1}\right\}$ and $U_{n+1} \times\{(n+1) /(n+2)\}$ are strong deformation retracts of $L_{n+1}$ and $W_{n+1}$ respectively, it follows that $r_{n+1}^{\prime \prime}=r_{n+1} \mid L_{n+1}: L_{n+1} \rightarrow W_{n+1}$ is a homotopy equivalence. By Theorem 1, we have homeomorphisms

$$
g: L_{n+1} \rightarrow W_{n+1} \quad \text { and } \quad g^{\prime}: \partial M_{n+1} \rightarrow U_{n+1} \times\left\{\frac{n+1}{n+2}\right\}
$$

such that $g \simeq r_{n+1}^{\prime \prime}$ and $g^{\prime} \simeq r_{n+1}^{\prime}=r_{n+1}^{\prime \prime} \mid \partial M_{n+1}$. Then $g^{\prime}$ extends to a homeomorphism

$$
g^{\prime \prime}: \bar{\partial} M_{n} \cup \partial M_{n+1} \rightarrow B_{n} \cup U_{n+1} \times\left\{\frac{n+1}{n+2}\right\}
$$

by setting $g^{\prime \prime} \mid \bar{\partial} M_{n}=g_{n}$.

Note that $r_{n}$ is homotopic to the map

$$
q_{n}: \bar{M}_{n} \rightarrow U_{n} \times\left[0, \frac{n}{n+1}\right]
$$

defined by $q_{n}(x)=\left(p_{n}(x), 0\right)$ and $\psi_{n} q_{n}=p_{n}$. Then we have $\psi_{n} \bar{f}_{n} \simeq \psi_{n} r_{n} \simeq$ $\psi_{n} q_{n}=p_{n}$. Let $c_{n}: \mathbf{I} \rightarrow\{n /(n+1)\}$ and $c_{n+1}: \mathbf{I} \rightarrow\{(n+1) /(n+2)\}$ be the constant maps. Since $r_{n+1}^{\prime \prime}\left|\bar{\partial} M_{n}=p_{n} \times c_{n+1}\right| \bar{\partial} M_{n}$, it follows that

$$
g_{n} \simeq \psi_{n} \bar{f}_{n} \times c_{n}\left|\bar{\partial} M_{n} \simeq p_{n} \times c_{n}\right| \bar{\partial} M_{n} \simeq p_{n} \times c_{n+1}\left|\bar{\partial} M_{n}=r_{n+1}^{\prime \prime}\right| \bar{\partial} M_{n},
$$

where all homotopies are realized in $W_{n+1}$ (the first two in $B_{n}$ ). Therefore,

$$
g^{\prime \prime} \simeq r_{n+1}^{\prime \prime}\left|\bar{\partial} M_{n} \cup \partial M_{n+1} \simeq g\right| \bar{\partial} M_{n} \cup \partial M_{n+1} .
$$

Thus, we can apply Theorem 2 to extend $g^{\prime \prime}$ to a homeomorphism $\tilde{g}: L_{n+1} \rightarrow$ $W_{n+1}$. By pasting $\tilde{g}$ with $\left(\psi_{n} \times\right.$ id $)\left(\bar{f}_{n} \times\right.$ id $)$, we can obtain the desired homeomorphism $\bar{f}_{n+1}$. Since $i_{n+1} p_{n+1} \simeq$ id in $\bar{M}_{n+1}$, it follows that

$$
\bar{f}_{n+1} \simeq \bar{f}_{n+1} i_{n+1} p_{n+1}=g^{\prime} i_{n+1} p_{n+1} \simeq r_{n+1} i_{n+1} p_{n+1} \simeq r_{n+1} .
$$

This completes the proof.

REMARK 2. For a closed tower $M_{1} \subset M_{2} \subset \cdots$ of $\ell_{2}(\tau)$-manifolds such that each $M_{i}$ is a $Z$-set in $M_{i+1}, M=\bigcup_{n \in \mathbb{N}}[n-1, n] \times M_{n}$ is an $\ell_{2}(\tau)$ manifold. On the other hand, given a sequence $\Psi=\left(\psi_{n}\right)_{n \in \mathbb{N}}$ of collars $\psi_{n}$ : $M_{n} \times[0,1) \rightarrow M_{n+1}$, the $\ell_{2}(\tau) \times \mathbb{R}^{\infty}$-manifold $M_{\Psi}$ can be defined as in Remark 1. Similarly to Lemma 3 , we can show $M \times \mathbb{R}^{\infty} \approx M_{\Psi}$. Since $M \times \mathbb{R}^{\infty}$ does not depend on $\Psi$, the topological type of $M_{\Psi}$ is unique. Moreover, $M_{\Psi}$ can be embedded in $\ell_{2}(\tau) \times \mathbb{R}^{\infty}$ as an open set.

\section{References}

[1] R. D. Anderson and J. D. McCharen, On extending homeomorphisms to Fréchet manifolds, Proc. Amer. Math. Soc. 25 (1970), 283-289. 
[2] C. Bessaga and A. Pełczyński, Selected Topics in Infinite-Dimensional Topology, Monogr. Mat. 58, Polish Sci. Publ., Warszawa, 1975.

[3] R. E. Heisey, Manifolds modelled on the direct limit of lines, Pacific J. Math. 102 (1982), 47-54.

[4] D. W. Henderson, Corrections and extensions of two papers about infinite-dimensional manifolds, General Topology Appl. 1 (1971), 321-327.

[5] D. W. Henderson and R. M. Schori, Topological classification of infinite-dimensional manifolds by homotopy type, Bull. Amer. Math. Soc. 76 (1970), 121-124.

[6] P. Mankiewicz, On topological, Lipschitz, and uniform classification of LF-spaces, Studia Math. 52 (1974), 109-142.

[7] K. Sakai, On $\mathbb{R}^{\infty}$-manifolds and $Q^{\infty}$-manifolds, Topology Appl. 18 (1984), 69-79.

[8] H. H. Schaefer with M. P. Wolff, Topological Vector Spaces, 2nd ed., Grad. Texts in Math. 3, Springer, New York, 1999.

[9] R. M. Schori, Topological stability of infinite-dimensional manifolds, Compos. Math. 23 (1971), 87-100.

[10] H. Toruńczyk, Absolute retracts as factors of normed linear spaces, Fund. Math. 86 (1974), 53-67.

[11] —, Characterizing Hilbert space topology, ibid. 111 (1981), 247-262.

[12] A. Wilansky, Modern Methods in Topological Vector Spaces, McGraw-Hill, New York, 1978.

Kotaro Mine and Katsuro Sakai

Institute of Mathematics

University of Tsukuba

Tsukuba, 305-8571, Japan

E-mail: pen@math.tsukuba.ac.jp

sakaiktr@sakura.cc.tsukuba.ac.jp

Received April 20, 2007

(7595) 